\title{
Estudio clínico de 200 pacientes con estomatitis aftosa recurrente
}

\author{
Alberto Rodríguez-Archilla y Tarik Raissouni \\ ${ }^{1}$ Universidad de Granada, Facultad de Odontología, Unidad de Medicina Bucal, Granada, España; ${ }^{2}$ Centro Odontológico Mfedal Afailal Bab Okla, \\ Tetuán, Marruecos
}

\section{Resumen}

Introducción: La estomatitis aftosa recurrente (EAR) afecta aproximadamente a $20 \%$ de la población general. Su etiología aún se desconoce. Objetivo: Analizar las características clínicas de esta entidad. Método: En 200 pacientes con EAR se analizó edad, sexo, historia familiar de EAR, edad de aparición del primer episodio, síntomas prodrómicos, número, tamaño, morfología y localización de las lesiones, forma clínica de EAR, tasa anual de recurrencia, factores predisponentes, sintomatología, tiempo de desaparición de los síntomas y las lesiones. Resultados: Los pacientes tenían formas menores de EAR. Las principales características clínicas fueron historia familiar de EAR (89\%), primer episodio con $\geq 10$ años de edad (69 \%), síntomas prodrómicos (66 \%), lesión por episodio (63\%), lesiones de < $0.5 \mathrm{~cm}$ (64\%), morfología redondeada (55\%), localización en lengua (27\%), tres episodios recurrentes anuales (36\%), estrés predisponente (34\%), desaparición de síntomas a los dos días (54\%) y curación de lesiones a los ocho días (40\%). Conclusión: Aun cuando la EAR es un trastorno común de la mucosa bucal, no se dispone de un tratamiento curativo. Las medidas terapéuticas buscan reducir el dolor y tamaño de las lesiones, acelerar el periodo de recuperación y disminuir la recidiva.

PALABRAS CLAVE: Enfermedades bucales. Estomatitis aftosa recurrente. Mucosa bucal.

\begin{abstract}
Introduction: Recurrent aphthous stomatitis (RAS) affects approximately $20 \%$ of the general population. Its etiology is still unknown. Objective: To analyze this entity's clinical features. Method: Data such as age, gender, family history of RAS, age at first episode onset, prodromal symptoms, number, size, morphology and localization of lesions, RAS clinical form, annual rate of recurrence, predisposing factors, symptoms and time for symptoms and lesions disappearance were assessed in 200 patients with RAS. Results: Patients had RAS minor forms. Main clinical characteristics were family history of RAS (89\%), first episode at $\geq 10$ years of age (69\%), prodromal symptoms (66\%), one lesion per episode (63\%), < $0.5 \mathrm{~cm}$ lesions (64\%), rounded morphology (55\%), localization at the tongue (27\%), 3 recurrent episodes per year (36\%), stress as predisposing factor (34\%), symptom disappearance in 2 days (54\%) and healing of lesions in 8 days (40\%). Conclusion: Even when RAS is a common disorder of the oral mucosa, there is no curative treatment available. Therapeutic measures seek to reduce the pain and size of lesions, accelerate the time of recovery and decrease the rate of relapses.
\end{abstract}

KEY WORDS: Mouth diseases. Recurrent aphthous stomatitis. Oral mucosa.

Fecha de recepción: 03-04-2016

Fecha de aceptación: 19-04-2016

DOI: 10.24875/GMM.18002503
Gac Med Mex. 2018;154:165-171

Disponible en PubMed www.gacetamedicademexico.com 


\section{Introducción}

La estomatitis aftosa recurrente (EAR) continúa siendo la enfermedad ulcerosa más común de la mucosa bucal. ${ }^{1}$ La EAR se caracteriza por la aparición de una o varias úlceras (aftas) dolorosas, inicialmente necróticas, cubiertas por una pseudomembrana blancas o grisáceas y rodeadas por un halo eritematoso bien definido. Las lesiones suelen afectar a la mucosa bucal no queratinizada, pueden persistir durante días o semanas y recurren después de periodos muy variables. ${ }^{2,3}$

La EAR afecta aproximadamente a $20 \%$ de la población general, aunque algunos estudios indican de 5 a $60 \%$, dependiendo del grupo étnico y socioeconómi$\mathrm{co}^{3,4}$ Son lesiones comunes en pacientes entre los 10 y 40 años de edad; tienen predilección por el sexo femenino y por individuos de nivel socioeconómico alto. ${ }^{5,6}$

La EAR es considerada un proceso multifactorial en el que se unen diversos factores desencadenantes y una alteración inmunológica de base. ${ }^{7}$ En el momento actual, la etiopatogenia de la EAR no es completamente conocida, aunque su aparición ha sido relacionada con diversos factores, destacando los factores genéticos con antecedentes familiares asociados con esta enfermedad. ${ }^{8}$ Otros factores relacionados son alergia alimentaria, trauma local, cambios hormonales (como los del ciclo menstrual), estrés y ansiedad, deficiencias nutricionales, agentes infecciosos y diversas enfermedades sistémicas. ${ }^{4,6,9,10}$ Las aftas son menos frecuentes en los individuos fumadores, lo que sugiere que el tabaco desempeña un posible papel protector. ${ }^{11}$

La respuesta inmunitaria en la EAR se caracteriza por estimulación antigénica de los queratinocitos, que favorece la activación de los linfocitos $T^{12,13}$ y la participación de inmunoglobulinas,${ }^{14}$ inmunocomplejos circulantes ${ }^{15}$ citocinas y moléculas de adhesión. ${ }^{16-18}$ Lo anterior da lugar a la acumulación de linfocitos y al desarrollo de una respuesta citotóxica que induce la aparición de úlceras en la mucosa bucal., ${ }^{6,9,19}$

Según el tamaño de las lesiones, se han descrito tres formas clínicas de EAR: aftas menores, aftas mayores y aftas herpetiformes. ${ }^{4,20}$

Las aftas menores son las más comunes (> $85 \%$ de los casos) y se caracterizan por lesiones superficiales de menos de $1 \mathrm{~cm}$ de diámetro, que aparecen normalmente en la mucosa bucal no queratinizada y curan en una a dos semanas sin dejar cicatriz.,20

Las aftas mayores son menos frecuentes $(10 \%$ de los casos) y presentan lesiones $>1 \mathrm{~cm}$ de diámetro, más profundas y muy dolorosas. Pueden aparecer en mucosa no queratinizada y en queratinizada. Requieren más de 2 semanas para curar y suelen hacerlo dejando cicatriz. ${ }^{4,20}$

Las aftas herpetiformes ( $5 \%$ de los casos) se caracterizan por episodios de múltiples lesiones superficiales de pequeño tamaño (2 a $3 \mathrm{~mm}$ ), agrupadas en forma de racimos y distribuidas por toda la mucosa no queratinizada. Las lesiones tienden a unirse y a formar úlceras irregulares de mayor tamaño. Por lo general curan entre siete a 10 días sin dejar cicatriz. Clínicamente pueden confundirse con herpes recurrente intraoral. 4,20

El diagnóstico de la EAR se basa en la historia clínica del paciente y en los hallazgos clínicos, pero no existen pruebas diagnósticas específicas. Debe tenerse en cuenta su asociación con algunas enfermedades sistémicas tales como la enfermedad de Behçet, neutropenia cíclica, enfermedad celíaca, síndrome PFAPA (fiebre periódica, adenitis, faringitis y aftas), síndrome MAGIC (úlcera orales y genitales con inflamación de cartílagos), síndrome de Sweet, enfermedad de Reiter 0 algunas inmunodeficiencias. ${ }^{21-23}$

La EAR es de origen desconocido, por lo que no se dispone de un tratamiento específico; las medidas terapéuticas básicamente están encaminadas a minimizar la sintomatología y prevenir las recurrencias. La elección de un tratamiento tópico o sistémico depende de la severidad de los síntomas, del tamaño, número de lesiones y frecuencia de episodios recurrentes. ${ }^{4}$

El objetivo de este estudio fue analizar las características clínicas de la EAR en una serie de pacientes que acudieron a recibir tratamiento a un consultorio odontológico.

\section{Método}

Fueron incluidos 200 pacientes que acudieron a recibir atención dental a un consultorio odontológico de Tetuán, Marruecos, entre enero de 2013 y enero de 2015. La detección de las lesiones fue un hallazgo casual durante la exploración oral. A todos se les proporcionó la información necesaria acerca de los propósitos de este trabajo, con el fin de obtener su consentimiento. La información fue incorporada a un registro clínico diseñado especialmente para este fin. El protocolo de estudio fue aprobado por el Comité de Ética de Investigación Humana de la Facultad de Odontología, Universidad de Granada (registro FOD-UGR-054/2012).

Fueron excluidos los pacientes que rechazaron participar en la investigación, que no firmaron el consentimiento informado, con hábito de fumar, que consumían alcohol habitualmente y aquellos con enfermedades 
sistémicas relacionadas con la aparición de úlceras bucales. Todos los pacientes fueron diagnosticados en el primer día de aparición de las lesiones.

A cada paciente se le aplicó un cuestionario que incluía edad, sexo, historia familiar de EAR, edad de aparición del primer episodio, síntomas prodrómicos, número, tamaño, morfología y ubicación de las lesiones en cada episodio, forma clínica de EAR, tasa de recurrencia (número de episodios/año), factores predisponentes, sintomatología del proceso y tiempo de desaparición de los síntomas y las lesiones.

Se realizó estadística descriptiva (media aritmética, desviación estándar y porcentajes) y estadística analítica para la comparación de variables, en la que se utilizaron pruebas paramétricas (t de Student, Anova) y no paramétricas ( $U$ de Mann-Whitney, Kruskal-Wallis, chi cuadrado y prueba de Fisher, cuando fue necesario). Se consideró como nivel mínimo de significación una $p<0.05$. Los datos fueron procesados con el programa estadístico SPSS Statistics versión 22.0 (IBM Corp., Armonk, NY, EE. UU.).

\section{Resultados}

De los 200 pacientes incluidos, 120 (60\%) eran mujeres y $80(40 \%)$ hombres, con una media de edad de $34.20 \pm 13.77$ años y un rango entre 10 y 74 años. No se encontraron diferencias estadísticamente significativas entre ambos grupos de población en cuanto a edad y sexo.

En la Tabla 1 se muestran las características clínicas de los 200 pacientes con EAR. Los pacientes refirieron historia familiar previa de EAR, tuvieron el primer episodio con una edad $\geq 10$ años, tenían síntomas prodrómicos, una única lesión por episodio, lesiones con un diámetro $<0.5 \mathrm{~cm}$, de morfología redondeada y la mayoría con localización en la lengua. Todos presentaron aftas menores, con una tasa de recurrencia de tres episodios por año; el estrés fue el factor predisponente más referido. Las lesiones eran dolorosas y en $20 \%$ de los pacientes coexistía impotencia funcional asociada; los síntomas desaparecieron a los dos días y la mayoría de las lesiones curaron al octavo día de su aparición.

Al comparar la edad con algunos parámetros clínicos del estudio (Tabla 2) se observó mayor edad media en los pacientes que tuvieron el primer episodio de EAR con $\geq 10$ años $(p<0.001)$, con lesiones de diámetro $>0.5 \mathrm{~cm}(p<0.01)$, de morfología irregular $(p<0.01)$, localizadas en el piso de la boca $(p<0.001)$ y con tasa anual de recurrencia $\geq 6$ episodios $(p<0.001)$, así como en quienes refirieron alergia alimentaria como factor predisponente $(p<0.05)$. Para el resto de los parámetros (historia familiar de EAR, síntomas prodrómicos, número de lesiones, sintomatología, desaparición de los síntomas o lesiones), las comparaciones con la edad no fueron estadísticamente significativas.

Cuando se analizó la influencia del sexo en algunos parámetros clínicos (Tabla 3), se encontraron más mujeres que hombres que tuvieron el primer episodio de EAR a edad $<10$ años $(p<0.001)$ y que presentaban lesiones de diámetro $<0.5 \mathrm{~cm}(p<0.05)$. En cambio, se hallaron más hombres que mujeres que referían estrés, alteraciones intestinales 0 alergia alimentaria como factores predisponentes $(p<0.01)$. Las restantes comparaciones no resultaron ser estadísticamente significativas.

Se observó una relación directa entre los síntomas prodrómicos experimentados por los pacientes y mayor número de lesiones por episodio $(p<0.001)$ o con las lesiones localizadas en la mucosa labial inferior $(p<0.001)$.

Respecto a la comparación entre el número de lesiones por episodio de EAR y algunos parámetros clínicos (Tabla 4) se encontró un número medio de lesiones mayores en los varones $(p<0.05)$, en pacientes que tuvieron el primer episodio de EAR con $\geq 10$ años ( $p$ $<0.001)$, con lesiones de diámetro $<0.5 \mathrm{~cm}(p<0.05)$ y con tasa de recurrencia anual continua $(p<0.05)$. Para el resto de los parámetros, las comparaciones con el número medio de lesiones por episodio no fueron estadísticamente significativas.

La relación entre el tamaño de las lesiones y algunos parámetros clínicos estudiados se presenta en la Tabla 5. Entre las lesiones pequeñas $(<0.5 \mathrm{~cm}$ de diámetro) destacaron las lesiones redondeadas $(p<0.001)$, localizadas en la lengua $(p<0.001)$ y las que curaron a los seis días de su aparición $(p<0.01)$. En cambio, entre las lesiones grandes ( 0.5 a $1 \mathrm{~cm}$ de diámetro) destacaron las lesiones ovaladas $(p<0.001)$, localizadas en el piso de la boca $(p<0.001)$ y las que curaron después de 10 días de su aparición $(p<0.01)$.

$\mathrm{Al}$ analizar la influencia de los factores predisponentes sobre el tiempo de desaparición de los síntomas o lesiones se observó que en la mitad de los pacientes que refirieron alergia alimentaria como factor predisponente, los síntomas desaparecieron a los tres días, encontrándose asociación estadísticamente significativa $(p<0.01)$. Las lesiones curaron después de 10 días en $72.5 \%$ de los pacientes, en quienes el estrés fue el factor predisponente, con una relación estadísticamente significativa $(p<0.001)$. 
Tabla 1. Principales características clínicas en 200 individuos con estomatitis aftosa recurrente

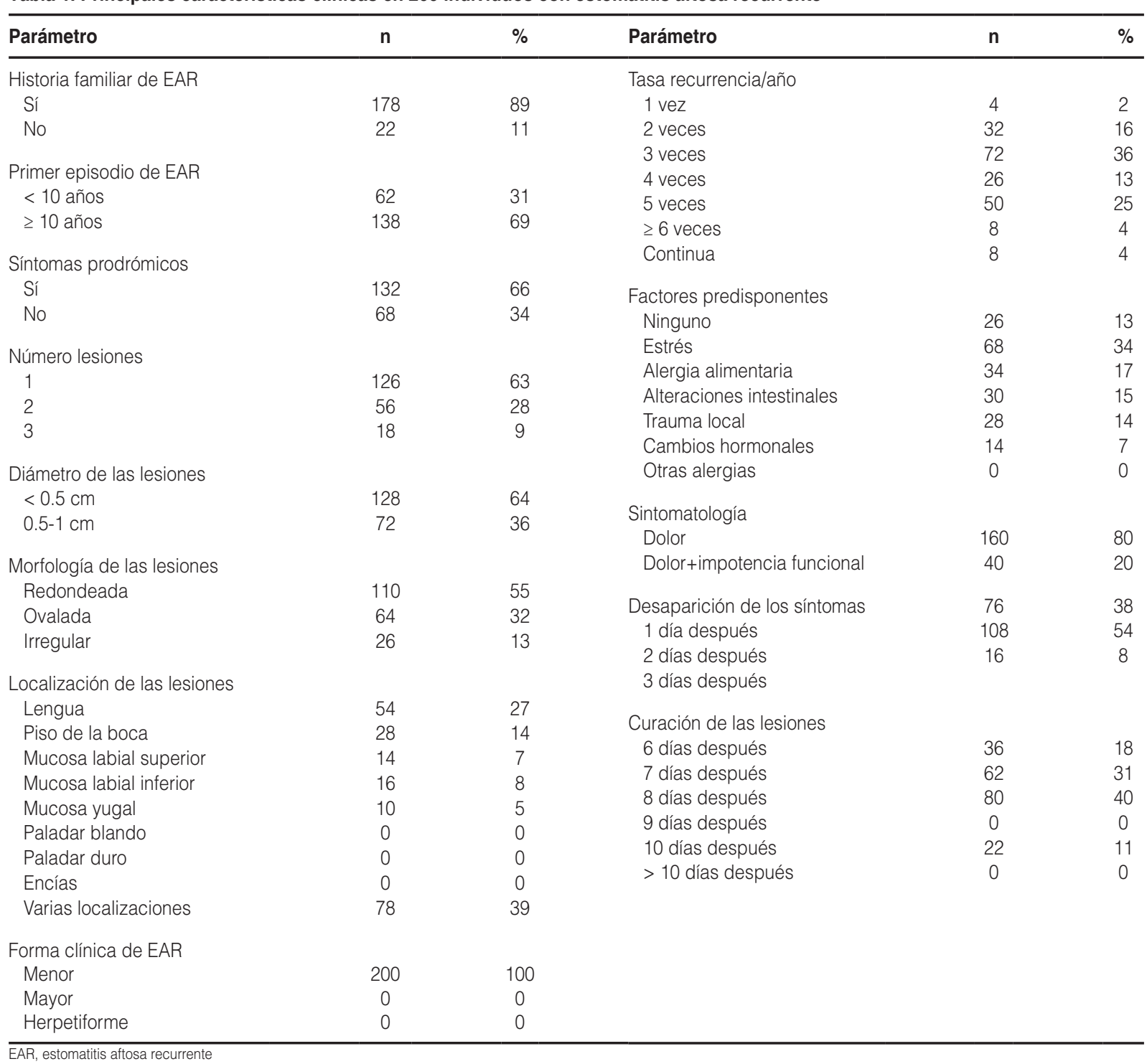

\section{Discusión}

La EAR es la enfermedad ulcerosa más frecuente de la mucosa bucal, a pesar de lo cual su etiología aún no se conoce del todo y no existe un tratamiento totalmente curativo. ${ }^{1}$

La población con EAR del presente estudio tenía una edad media de $34.20 \pm 13.77$ años, con un rango entre 10 y 74, que coincide con la información obtenida por Chattopadhyay et al. ${ }^{24}$ en un amplio estudio en 20050 adultos norteamericanos con EAR, en quienes la edad media fue de $36.10 \pm 1.92$ años. En Jordania, los pacientes incluidos en el trabajo de Safadi ${ }^{25}$ tenían una edad media de 37.5 años, con un rango de 13 a 68 . Por su parte, Brailo et al. ${ }^{26}$ registraron una edad media de 38.8 años, rango de 5 a 73 , en 68 pacientes croatas.
Oh et al. ${ }^{27}$ hallaron una edad media de 40.75 años en 1643 pacientes chinos. Otros estudios han registrado edades medias de $41.77 \pm 20.71$ años, rango de 21 a 65 años; ${ }^{28} 43.08 \pm 8.37$ años, rango de 21 a $65 ;{ }^{29} 42.80$ \pm 14.30 años, rango de 17 a $79 .{ }^{30}$ Aunque la EAR es más frecuente en jóvenes que en adultos, ${ }^{4,24}$ en este estudio fue el contrario: $27 \%$ de los pacientes se situó en el intervalo entre 20 a 30 años, mientras que $17 \%$ se encontraba en el intervalo entre 10 y 20 años.

La mayoría de los autores $3,7,25,27-29,31$ coincide en señalar que la EAR afecta con mayor frecuencia a las mujeres, sin embargo, en algunos estudios ${ }^{2,32}$ la prevalencia fue igual para ambos sexos, como en el de Majorana et al..$^{33}$ en niños italianos < 12 años, en quienes se observó ligero predominio del sexo masculino. En concordancia con lo expuesto en nuestro trabajo, la EAR fue más frecuente en las mujeres (60\%). 
Tabla 2. Influencia de la edad sobre parámetros clínicos en $\mathbf{2 0 0}$ individuos con estomatitis aftosa recurrente

\begin{tabular}{|c|c|c|c|}
\hline Parámetro & $\begin{array}{l}\text { Pacientes } \\
\text { (n) }\end{array}$ & $\begin{array}{c}\text { Edad } \\
(\bar{X} \pm D E)\end{array}$ & $\mathrm{p}$ \\
\hline \multicolumn{4}{|c|}{ Edad al primer episodio (años) } \\
\hline$<10$ & 62 & $27.65 \pm 9.88$ & $<0.001$ \\
\hline$\geq 10$ & 138 & $37.14 \pm 14.27$ & \\
\hline \multicolumn{4}{|l|}{ Tamaño lesiones } \\
\hline Pequeñas $(<0.5 \mathrm{~cm})$ & 128 & $32.27 \pm 12.80$ & $<0.01$ \\
\hline Grandes $(0.5-1 \mathrm{~cm})$ & 72 & $37.64 \pm 14.82$ & \\
\hline \multicolumn{4}{|l|}{ Morfología de las lesiones } \\
\hline Redondeada-ovalada & 174 & $32.84 \pm 13.43$ & $<0.01$ \\
\hline Irregular & 26 & $43.31 \pm 12.70$ & \\
\hline \multicolumn{4}{|l|}{ Localización de las lesiones } \\
\hline Piso de la boca & 28 & $47.43 \pm 13.97$ & $<0.001$ \\
\hline Resto de localizaciones & 172 & $32.05 \pm 12.51$ & \\
\hline \multicolumn{4}{|l|}{ Tasa anual de recurrencia } \\
\hline 1 vez & 4 & $18.50 \pm 8.66$ & $<0.05$ \\
\hline Resto & 196 & $34.52 \pm 13.68$ & \\
\hline \multicolumn{4}{|l|}{ Factores predisponentes } \\
\hline Alergia alimentaria & 34 & $38.65 \pm 15.27$ & $<0.05$ \\
\hline Resto de factores & 166 & $33.29 \pm 13.31$ & \\
\hline
\end{tabular}

Tabla 3. Relación de parámetros clínicos con el sexo en 200 individuos con estomatitis aftosa recurrente

\begin{tabular}{|c|c|c|c|c|c|}
\hline \multirow[t]{3}{*}{ Parámetro } & \multicolumn{4}{|c|}{ Sexo } & \multirow[t]{3}{*}{$\mathrm{p}$} \\
\hline & \multicolumn{2}{|c|}{ Masculino } & \multicolumn{2}{|c|}{ Femenino } & \\
\hline & $\mathbf{n}$ & $\%$ & $\mathbf{n}$ & $\%$ & \\
\hline Edad al primer episodio (años) & & & & & $<0.01$ \\
\hline$<10$ & 16 & 20.0 & 46 & 38.3 & \\
\hline$\geq 10$ & 64 & 80.0 & 74 & 61.7 & \\
\hline \multicolumn{6}{|l|}{ Tamaño lesiones (diámetro) } \\
\hline Pequeñas $(<0.5 \mathrm{~cm})$ & 44 & 55.0 & 84 & 70.0 & $<0.05$ \\
\hline Grandes $(0.5-1 \mathrm{~cm})$ & 36 & 45.0 & 36 & 30.0 & \\
\hline \multicolumn{6}{|l|}{ Factores predisponentes } \\
\hline Ninguno & 8 & 10.0 & 18 & 15.0 & \\
\hline Estrés & 30 & 37.5 & 38 & 31.7 & \\
\hline Alteraciones intestinales & 16 & 20.0 & 14 & 11.6 & $<0.01$ \\
\hline Trauma local & 8 & 10.0 & 20 & 16.7 & \\
\hline Alergia alimentaria & 18 & 22.5 & 16 & 13.3 & \\
\hline Cambios hormonales & 0 & 0.0 & 14 & 11.7 & \\
\hline
\end{tabular}

Un factor condicionante para el desarrollo de EAR es la existencia de antecedentes familiares. ${ }^{25,31,34} \mathrm{De}$ hecho, los pacientes con antecedentes familiares tuvieron $90 \%$ de probabilidades de desarrollar EAR, en contraposición con $20 \%$ de los pacientes sin antecedentes familiares. ${ }^{4}$ En la investigación que se presenta, $89 \%$ de los pacientes refirió historia familiar de EAR.

Algunas investigaciones ${ }^{3,7,35}$ sitúan el primer episodio de EAR en la segunda década de la vida, coincidiendo con el inicio de la pubertad, hecho que concuerda con los resultados obtenidos: $69 \%$ de los pacientes tuvo el primer episodio entre los 10 y 20 años versus $31 \%$ en quienes el primer episodio apareció antes de los 10 años.

En cuanto al número de lesiones, diversos autores ${ }^{1,2,36-38}$ indican que los episodios de EAR cursan con menos de tres lesiones en la mayoría de las ocasiones. En este trabajo, $63 \%$ de los pacientes tuvo episodios con una única lesión. Safadi, ${ }^{25}$ en su estudio sobre 561 pacientes, observó $49.2 \%$ de pacientes con una sola lesión por episodio. El número de lesiones por episodio estuvo condicionado por el sexo, la edad de aparición del primer episodio, el tamaño de las lesiones y la tasa anual de recurrencia.

Casi todas las investigaciones coinciden en relacionar el tamaño de las lesiones con la forma clínica de la EAR, ${ }^{1,2,4,7,19,20,35}$ diferenciando entre formas menores (diámetro $<1 \mathrm{~cm}$ ), mayores (diámetro $>1 \mathrm{~cm}$ ) y herpetiformes (2 a $3 \mathrm{~mm}$ ). En este estudio, todas las lesiones de EAR eran menores, que en términos generales son las más frecuentes ( $85 \%$ de los casos)., ${ }^{4,20}$

Respecto a la forma de las lesiones, la mayoría de Ios autores ${ }^{2,3,7,19,32,36,37,39}$ distingue entre lesiones irregulares y regulares (ovaladas o redondeadas); estas últimas son las más frecuentes, asociadas a formas menores de EAR. Los resultados del presente trabajo (87\% de lesiones con morfología regular y $13 \%$ con irregular), coinciden con los publicados por otros investigadores. Los registros de Safadi ${ }^{25}$ indican $34.3 \%$ de lesiones irregulares y $65.7 \%$ de regulares, lo que discrepa de nuestros hallazgos, si bien más de $15 \%$ de sus pacientes tenía formas mayores de EAR.

Numerosos estudios ${ }^{17,9,19,32,35}$ concuerdan en indicar una predilección de las lesiones de EAR por localizarse en áreas de mucosa bucal no queratinizada. De forma análoga, en este estudio la localización más frecuente de las lesiones fue la lengua con $27 \%$ de los casos, seguida de la mucosa labial interna (15\%), piso de la boca (14 \%) o mucosa yugal (5\%). Ningún caso se localizó en el paladar blando o en la mucosa bucal queratinizada (paladar duro, encías). También refieren la lengua como la localización más frecuente de las lesiones de EAR, los trabajos de Safadi, ${ }^{25}$ en Jordania, Oh, et al.., ${ }^{27}$ en China o los de López-Jornet et al., ${ }^{28}$ en España. En cambio, otros ${ }^{1,2,31,40}$ sitúan a la mucosa labia interna como la localización más frecuente por delante de la lengua.

En el presente trabajo, la tasa anual de recurrencia fue de tres episodios (36\%), seguida de cinco episodios $(25 \%$ ), dos episodios (16\%) y solo $4 \%$ de los pacientes tenía aftas de forma continua. En consonancia con estos resultados, Axell y Henricsson, ${ }^{40}$ en un 
estudio sobre 3500 pacientes, sólo encontraron $1 \%$ de recurrencia continua, $15 \%$ de afectación frecuente y $84 \%$ de clínica intermitente, es decir, pacientes que tenían menos de seis episodios por año. En sentido opuesto, Bagán et al., ${ }^{38}$ al analizar una serie de 93 pacientes observaron que $53.8 \%$ presentaba una tasa anual de recurrencia de cinco a ocho episodios, superior a las encontrada en nuestro estudio. Las aparentes discrepancias entre los diversos autores en cuanto a la tasa de recurrencia anual de la EAR pueden atribuirse a los diferentes tamaños de muestra y al establecimiento de intervalos de recurrencia diferentes en los diseños metodológicos de los estudios.

Tabla 4. Relación entre el número medio de lesiones y algunos parámetros clínicos en $\mathbf{2 0 0}$ individuos con estomatitis aftosa recurrente

\begin{tabular}{lccc}
\hline Parámetro & $\mathbf{n}$ & $\begin{array}{c}\text { Número de lesiones } \\
\text { (media) }\end{array}$ & $\mathbf{p}$ \\
\hline $\begin{array}{l}\text { Sexo } \\
\quad \text { Masculino }\end{array}$ & 80 & $1.58 \pm 0.70$ & $<0.05$ \\
$\quad$ Femenino & 120 & $1.38 \pm 0.61$ & \\
Edad al primer episodio (años) & & & $<0.01$ \\
$\quad<10$ & 62 & $1.26 \pm 0.51$ & \\
$\quad 10$ & 138 & $1.55 \pm 0.69$ & \\
$\quad$ & & & $<0.05$ \\
Tamaño lesiones & 128 & $1.53 \pm 0.68$ & \\
$\quad$ Pequeñas $(<0.5 \mathrm{~cm})$ & 72 & $1.33 \pm 0.58$ & \\
$\quad$ Grandes $(0.5-1 \mathrm{~cm})$ & & & \\
$\quad$ Tasa de recurrencia & 8 & $2.00 \pm 1.07$ & $<0.05$ \\
$\quad$ Continua & 192 & $1.44 \pm 0.63$ & \\
$\quad$ Resto de tasas & & & \\
\hline
\end{tabular}

Se han analizado diversos factores predisponentes relacionados con la EAR. En el presente estudio, los tres más importantes fueron el estrés (34\%), la alergia alimentaria (17\%) y las alteraciones intestinales (15\%). El estrés como factor predisponente relevante de la EAR ha sido ampliamente sugerido en investigaciones que consideran a la mucosa oral como un órgano diana en determinados desórdenes emocionales. ${ }^{2,3,19,25,30,32,38,41}$ La disparidad de los resultados en torno a este factor probablemente radica en los tamaños de muestra estudiados, el tipo de población y las diferencias en el diseño metodológico de las investigaciones.

La alergia alimentaria como factor precipitante de la EAR también ha sido mencionada. ${ }^{25,42,43}$ Incluso, hay análisis en los que llega a ser el factor predisponente más importante: 50 o $75 \%$ de los casos. ${ }^{41,43}$ Dado el pequeño tamaño muestral de esas investigaciones, los resultados deben considerarse poco representativos. Además, deben considerarse otros factores particulares en cada paciente, que actúan mediante un mecanismo desconocido. Se requieren estudios más amplios que profundicen en este aspecto y permitan aclarar las divergencias.

Las alteraciones intestinales, ya sea por deficiencias nutricionales o patologías gastrointestinales, también han sido citadas como causas desencadenantes en diversos trabajos. ${ }^{1,25.28,44}$

Los cambios hormonales constituyeron el factor predisponente menos frecuente $(7 \%)$, en coincidencia con lo informado por Axéll et al. ${ }^{40}(8 \%)$ y Lotufo et al. ${ }^{41}(2.5 \%)$. En una revisión sistemática realizada

Tabla 5. Relación entre el tamaño de las lesiones y algunos parámetros en 200 individuos con estomatitis aftosa recurrente

\begin{tabular}{|c|c|c|c|c|c|}
\hline \multirow[t]{3}{*}{ Parámetro } & \multicolumn{4}{|c|}{ Tamaño de las lesiones } & \multirow[t]{3}{*}{$\mathrm{p}$} \\
\hline & \multicolumn{2}{|c|}{ Pequeñas $<0.5 \mathrm{~cm}$ ) } & \multicolumn{2}{|c|}{ Grandes $0.5-1 \mathrm{~cm}$ ) } & \\
\hline & $\mathrm{n}$ & $\%$ & $\mathrm{n}$ & $\%$ & \\
\hline \multicolumn{6}{|l|}{ Morfología lesiones } \\
\hline Redondeada & 84 & 65.6 & 26 & 36.1 & $<0.001$ \\
\hline Ovalada & 34 & 26.6 & 30 & 41.7 & \\
\hline Irregular & 10 & 7.8 & 16 & 22.2 & \\
\hline \multicolumn{6}{|l|}{ Localización lesiones } \\
\hline Lengua & 40 & 31.3 & 14 & 19.4 & \\
\hline Piso de la boca & 4 & 3.1 & 24 & 33.3 & \\
\hline Mucosa labial superior & 12 & 9.4 & 2 & 2.8 & $<0.001$ \\
\hline Mucosa labial inferior & 10 & 7.8 & 6 & 8.3 & \\
\hline Mucosa yugal & 6 & 4.7 & 4 & 5.6 & \\
\hline Varias localizaciones & 56 & 43.8 & 22 & 30.6 & \\
\hline \multicolumn{6}{|c|}{ Curación lesiones (días después) } \\
\hline 6 & 30 & 83.3 & 6 & 8.3 & \\
\hline 7 & 36 & 58.1 & 26 & 41.9 & $<0.01$ \\
\hline 8 & 54 & 67.5 & 26 & 32.5 & \\
\hline 10 & 8 & 36.4 & 14 & 63.6 & \\
\hline
\end{tabular}


por McCartan y Sullivan ${ }^{45}$ no se encontró relación entre la aparición de EAR, ciclo menstrual, embarazo, menopausia o terapia hormonal sustitutiva; en cambio, algunos autores ${ }^{3,7,27,39}$ han otorgado una relevancia mucho mayor a este factor. Las diferencias en este apartado podrían deberse a los distintos tamaños de muestra, diferencias en la distribución por sexo y planteamientos de las investigaciones.

En este estudio en pacientes con formas menores de EAR, la mayoría tenía historia familiar de EAR, episodios de una lesión única, de morfología regular (ovalada o redondeada), localizada principalmente en la lengua o mucosa labial interna y con una tasa de recurrencia anual de tres episodios. El factor desencadenante más relacionado fue el estrés, el tiempo de desaparición de la sintomatología fue de dos días y el de remisión de 8 días a partir del brote.

Aun cuando la EAR es uno de los trastornos más comunes de la mucosa bucal y que sus características clínicas están bien definidas, su etiología y patogenia precisas siguen sin ser claras y, por lo tanto, no se dispone de un tratamiento curativo. Las medidas terapéuticas están orientadas a reducir el dolor y el tamaño de las lesiones, acelerar el periodo de curación y disminuir la frecuencia de recidiva, por lo que se requieren nuevos trabajos que profundicen en el estudio de los mecanismos etiopatogénicos de esta enfermedad y de sus factores desencadenantes, con el fin de conseguir un tratamiento totalmente efectivo.

\section{Bibliografía}

1. Porter SR, Scully C, Pedersen A. Recurrent aphthous stomatitis. Crit Rev Oral Biol Med. 1998;9(3):306-21.

2. Ship JA, Chavez EM, Doerr PA, Henson BS, Sarmadi M. Recurrent aphthous stomatitis. Quintessence Int. 2000;31(2):95-112.

3. Jurge S, Kuffer R, Scully C, Porter SR. Mucosal disease series. Number VI. Recurrent aphthous stomatitis. Oral Dis. 2006;12(1):1-21.

4. Akintoye SO, Greenberg MS. Recurrent aphthous stomatitis. Dent Clin North Am. 2014;58(2):281-297.

5. Baccaglini L, Lalla RV, Bruce AJ, Sartori-Valinotti JC, Latortue MC, Carrozzo M, et al. Urban legends: recurrent aphthous stomatitis. Oral Dis. 2011;17(8):755-770.

6. Chavan M, Jain H, Diwan N, Khedkar S, Shete A, Durkar S. Recurrent aphthous stomatitis: a review. J Oral Pathol Med. 2012;41(8):577-583.

7. Porter SR, Hegarty A, Kaliakatsou F, Hodgson TA, Scully C. Recurrent aphthous stomatitis. Clin Dermatol. 2000;18(5):569-578.

8. Albanidou-Farmaki E, Deligiannidis A, Markopoulos AK, Katsares V Farmakis K, Parapanissiou E. HLA haplotypes in recurrent aphthous stomatitis: a mode of inheritance? Int J Immunogenet. 2008;35(6):427-432.

9. Preeti L, Magesh KT, Rajkumar K, Karthik R. Recurrent aphthous stomatitis. J Oral Maxillofac Pathol. 2011;15(3):252-256.

10. Huling LB, Baccaglini L, Choquette L, Feinn RS, Lalla RV. Effect of stressful life events on the onset and duration of recurrent aphthous stomatitis. J Oral Pathol Med. 2012;41(2):149-152.

11. McRobbie $H$, Hajek $P$, Gillison $F$. The relationship between smoking cessation and mouth ulcers. Nicotine Tob Res. 2004;6(4):655-659.

12. Landesberg $R$, Fallon $M$, Insel R. Alterations of $T$ helper/inducer and $T$ suppressor/inducer cells in patients with recurrent aphthous ulcers. Oral Surg Oral Med Oral Pathol. 1990;69(2):205-208.

13. Bachtiar EW, Cornain S, Siregar B, Raharjo TW. Decreased CD4+/ CD8+ ratio in major type of recurrent aphthous ulcers: comparing major to minor types of ulcers. Asian Pac J Allergy Immunol. 1998; 16(2-3):75-79.
14. Scully C, Yap PL, Boyle P. IgE and IgD concentrations in patients with recurrent aphthous stomatitis. Arch Dermatol. 1983;119(1):31-34.

15. Rodríguez-Archilla A, Urquía M, Gómez-Moreno G, Ceballos A. Anti-DNA antibodies and circulating immune complexes (C1q-lgG) in recurrent aphthous stomatitis. Bull Group Int Rech Sci Stomatol Odontol. 1994;37(1-2):31-35.

16. Yamamoto T, Yoneda K, Ueta E, Osaki T. Serum cytokines, interleukin-2 receptor, and soluble intercellular adhesion molecule-1 in oral disorders. Oral Surg Oral Med Oral Pathol. 1994;78(6):727-35.

17. Healy CM, Enobakhare B, Haskard DO, Thornhill MH. Raised levels of circulating VCAM-1 and circulating E-selectin in patients with recurrent oral ulceration. J Oral Pathol Med. 1997;26(1):23-28.

18. Healy CM, Thornhill MH. Induction of adhesion molecule expression on blood vessels and keratinocytes in recurrent oral ulceration. J Oral Pathol Med. 1999;28(1):5-11.

19. Scully C, Porter S. Oral mucosal disease: recurrent aphthous stomatitis. Br J Oral Maxillofac Surg. 2008;46(3):198-206.

20. Scully C. Clinical practice. Aphthous ulceration. N Engl J Med. 2006;355(2):165-172.

21. Taylor J, Glenny AM, Walsh T, Brocklehurst P, Riley P, Gorodkin R, et al. Interventions for the management of oral ulcers in Behçet's disease. Cochrane Database Syst Rev. 2014;(9):CD011018.

22. Maloney WJ, Raymond G, Hershkowitz D, Rochlen G. Oral and dental manifestations of celiac disease. N Y State Dent J. 2014;80(4):45-48.

23. Burton MJ, Pollard AJ, Ramsden JD, Chong LY, Venekamp RP. Tonsillectomy for periodic fever, aphthous stomatitis, pharyngitis and cervical adenitis syndrome (PFAPA). Cochrane Database Syst Rev. 2014;(9):CD008669.

24. Chattopadhyay A, Chatterjee S. Risk indicators for recurrent aphthous ulcers among adults in the US. Community Dent Oral Epidemiol. 2007;35(2):152-159.

25. Safadi RA. Prevalence of recurrent aphthous ulceration in Jordanian dental patients. BMC Oral Health. 2009;9:31.

26. Brailo V, Boras VV, Cekić-Arambasin A. Rekurentne aftozne ulceracije: Analiza predisponirajucih cimbenika u 68 bolesnika. Liječ Vjesn. 2007;129:4-7.

27. Oh SH, Han EC, Lee JH, Bang D. Comparison of the clinical features of recurrent aphthous stomatitis and Behçet's disease. Clin Exp Dermatol. 2009;34(6):e208-e212.

28. López-Jornet $\mathrm{P}$, Camacho-Alonso F, Martos N. Hematological study of patients with aphthous stomatitis. Int J Dermatol. 2014;53(2):159-163.

29. Gavic L, Cigic L, Biocina-Lukenda D, Gruden V, Gruden Pokupec JS. The role of anxiety, depression, and psychological stress on the clinical status of recurrent aphthous stomatitis and oral lichen planus. J Oral Pathol Med. 2014;43(6):410-417.

30. Bratel J, Hakeberg M. Anamnestic findings from patients with recurrent aphthous stomatitis. Swed Dent J. 2014;38(3):143-149.

31. McCullough MJ, Abdel-Hafeth S, Scully C. Recurrent aphthous stomatitis revisited; clinical features, associations, and new association with infant feeding practices? J Oral Pathol Med. 2007;36(10):615-620.

32. Casiglia JM. Recurrent aphthous stomatitis: etiology, diagnosis and treatment. Gen Dent. 2002;50(2):157-166

33. Majorana A, Bardellini E, Flocchini P, Amadori F, Conti G, Campus G. Oral mucosal lesions in children from 0 to 12 years old: ten years' experience. Oral Surg Oral Med Oral Pathol Oral Radiol Endod. 2010;110(1):e13-e18.

34. Slebioda Z, Szponar E, Kowalska A. Etiopathogenesis of recurrent aphthous stomatitis and the role of immunologic aspects: literature review. Arch Immunol Ther Exp (Warsz). 2014;62(3):205-215.

35. Murray LN, Amedee RG. Recurrent aphthous stomatitis. J La State Med Soc. 2000;152(1):10-14.

36. Piantanida EW, Samlaska CP. Recurrent aphthous stomatitis. Oral Surg Oral Med Oral Pathol Oral Radiol Endod. 1996;82(5):472.

37. Rees TD, Binnie WH. Recurrent aphthous stomatitis. Dermatol Clin. 1996;14(2):243-256.

38. Bagán JV, Sanchis JM, Milián MA, Peñarrocha M, Silvestre FJ. Recurrent aphthous stomatitis. A study of the clinical characteristics of lesions in 93 cases. J Oral Pathol Med. 1991;20(8):395-397.

39. Zain RB. Oral recurrent aphthous ulcers/stomatitis: prevalence in Malaysia and an epidemiological update. J Oral Sci. 2000;42(1):15-19.

40. Axéll $T$, Henricsson V. The occurrence of recurrent aphthous ulcers in an adult Swedish population. Acta Odontol Scand. 1985;43(2):121-125.

41. Lotufo MA, Lemos CA, Shimizu MT, Cabral R, Birman EG. Clinical evaluation of the topical use of propolis in recurrent minor aphthous ulceration. Cienc Odontol Bras. 2005;8(3):6-9.

42. Ogura M, Yamamoto T, Morita M, Watanabe T. A case-control study on food intake of patients with recurrent aphthous stomatitis. Oral Surg Oral Med Oral Pathol Oral Radiol Endod. 2001;91(1):45-49.

43. Nolan A, Lamey PJ, Milligan KA, Forsyth A. Recurrent aphthous ulceration and food sensitivity. J Oral Pathol Med. 1991;20(10):473-475.

44. Koybasi S, Parlak AH, Serin E, Yilmaz F, Serin D. Recurrent aphthous stomatitis: investigation of possible etiologic factors. Am J Otolaryngol. 2006;27(4):229-232.

45. McCartan BE, Sullivan A. The association of menstrual cycle, pregnancy, and menopause with recurrent oral aphthous stomatitis: a review and critique. Obstet Gynecol. 1992;80(3 Pt 1):455-458. 